\title{
Repeated Onabotulinumtoxin-A Injections Provide Better Results than Single Injection in Treatment of Painful Bladder Syndrome
}

Hann-Chorng Kuo, MD

From: Department of Urology, Buddhist Tzu Chi General Hospital and Tzu Chi University, Hualien,

Taiwan.

Address Correspondence:

Dr. Hann-Chorng Kuo

Department of Urology Buddhist Tzu Chi General Hospital 707, Section 3, Chung Yang Road Hualien, Taiwan

E-mail: hck@tzuchi.com.tw

Disclaimer: There was no external funding in the preparation of this manuscript.

Conflict of interest: None.

Manuscript received: 06/19/2012 Revised manuscript received: 08/14/2012

Accepted for publication $09 / 20 / 2012$

Free full manuscript: www.painphysicianjournal.com
Background: Onabotulinumtoxin-A (BoNT-A) is effective for the treatment of interstitial cystitis/painful bladder syndrome (IC/PBS). However, long-term follow-up does not show successful outcome after a single injection.

Objectives: To evaluate the efficacy and safety of repeated intravesical BoNT-A injections for treatment of IC/PBS and compare the success rates among patient groups receiving different injection numbers.

Study Design: Prospective interventional study.

Setting: Tertiary medical center.

Methods: Intravesical injection of $100 \mathrm{U}$ of BoNT-A was performed in 81 patients every 6 months for up to 4 times or until patients' symptoms significantly improved. Patients who received a single injection served as active controls. Measured parameters included O'LearySant symptom indexes (ICSI) and problem indexes (ICPI), visual analogue score (VAS) for pain, voiding diary variables, urodynamic parameters, maximal bladder capacity under anesthesia, glomerulation grade, and global response assessment. Multiple measurements and KaplanMeier analysis were used for comparison of consecutive data and success rates among groups.

Results: Among 81 patients, 20 received single injections, 19 received 2 injections, 12 received 3 injections, and 30 received 4 injections. The mean ( \pm standard deviation) of ICSI, ICPI, total scores, VAS, functional bladder capacity, and daytime frequency all showed significant improvement after repeated BoNT-A treatment with different injections. Significantly better success rates were noted in patients who received 4 repeated injections $(P=0.0242)$ and 3 injections $(P=0.050)$, compared to those who received a single injection. However, there was no significant difference of long-term success rates among patients who received 2,3 , and 4 injections.

Limitations: Lack of placebo control group is the main limitation.

Conclusion: Repeated intravesical BoNT-A injections were safe and effective for pain relief and they increased bladder capacity and provided a better long-term success rate than a single injection did for treatment of IC/PBS.

Key words: Botulinum toxin, IC/PBS, Intravesical treatment

Pain Physician 2013; 16:E15-E23 
nterstitial cystitis/bladder pain syndrome (IC/BPS) is characterized by bladder pain associated with urgency, frequency, nocturia, dysuria, and sterile urine. The diagnosis of this disease remains unclear and should be based on exclusion of other diseases. The etiology of IC/BPS is thought to be multi-factorial, multiple therapies might produce synergistic effects and a better outcome. However, current treatments usually cannot completely eradicate bladder pain and increasing bladder capacity (1).

Previous studies demonstrated that urothelium plays an important barrier function in urine and bladder tissue (2). Denudation, or thinning of the bladder epithelium, is found in bladder mucosal biopsies from IC/PBS patients, suggesting altered regulation of urothelial homeostasis. Increased urothelial apoptosis is also noted and could be responsible for the loss of epithelial integrity (3). The suburothelial space immediately below the basal lamina is rich in sensory nerves which may respond to bladder inflammation $(4,5)$. Chronic suburothelial inflammation might inhibit normal basal cell proliferation and affect apical urothelial function (3). Treatment of urothelial dysfunction cannot be based solely on replacement of defense glycoproteins in the bladder urothelium. Injection of botulinum toxin type A (onabotulinumtoxin-A, BoNT-A) into the detrusor muscles increased bladder capacity and compliance in a chemical cystitis rat model (6). For patients who are refractory to oral medication or intravesical glycosaminoglycan replenishment therapies, evidence has shown that intravesical injections of BoNT-A might provide short-term symptomatic relief (7).

BoNT-A is an inhibitor of acetylcholine release at the presynaptic neuromuscular junction. Inhibition of acetylcholine release results in regional decreased muscle contractility at the injection sites. (8-11). BoNT-A is widely reported as effective for the treatment of neurogenic and idiopathic detrusor overactivity $(12,13)$; however, the application of BoNT-A to IC/PBS was reported in only a few studies (14-16). BoNT-A might not only reduce bladder sensitivity in IC/BPS patients but also induce desensitization in the central nervous system through affecting the over-expression of activated proteins in the dorsal horn ganglia in animal and human experiments $(8,9,15-17)$. Although BoNT-A injection provides symptomatic relief in treating IC/PBS, long-term therapeutic effects have not been achieved after single injections (18). The limited long-term success is possibly due to the poorly sustained therapeutic effect of a single BoNT-A injection on chronic bladder inflammation.
The purpose of this study is to evaluate the efficacy and safety of repeated intravesical BoNT-A injections in comparison with a single injection for the treatment of IC/PBS refractory to conventional treatment.

\section{Methods}

This study was approved by the Institutional Review Board (IRB) of the Tzu-Chi General Hospital (TCGH 100-06).

\section{Patient Enrollment}

Eighty-one consecutive patients with IC/PBS who failed conventional treatments were prospectively enrolled in this study from July 2006 to August 2010. A diagnosis of IC/PBS was established based on characteristic symptoms and cystoscopic findings of glomerulations, petechia, mucosal fissure, or ulceration (19). All patients had been treated with at least one of the following medications: oral pentosanpolysulphate, intravesical instillation of heparin, hyaluronic acid, or tricyclic antidepressant for more than one year, but the symptoms remained unchanged or had relapsed. They were investigated thoroughly on enrollment and were excluded if they did not meet the inclusion criteria of the National Institute of Diabetes Digestive and Kidney Diseases (NIDDK) (20).

\section{Clinical Assessment}

Careful history taking, physical examination, symptom assessment, questionnaires, and a 3-day voiding diary were performed in all patients on an outpatient basis. Urinalysis, urinary cultures, and urinary cytology were performed in all enrolled patients to exclude urinary tract infections (UTI) and bladder cancer. The ICI PBS symptoms were assessed using the O'Leary-Sant symptom indexes (ICSI) and problem indexes (ICPI) (21). The pain score was reported by self-assessment using a 10-point visual analog scale (VAS) system. Videourodynamic studies and potassium chloride $(\mathrm{KCl})$ sensitivity testing were performed. Additionally, patients were informed of the possible complications associated with BoNT-A injection such as generalized muscle weakness, difficult urination, transient urinary retention, or UTI. The primary end-point was the change in the sum of the ICSI and ICPI from baseline to 6 months after the last treatment.

The treatment outcomes were assessed using the global response assessment (GRA) $(22,23)$. Patients were requested to rate their bladder symptoms compared with baseline on a 7-point centered scale from 
markedly (-3), moderately (-2), and slightly worse $(-1)$, no change (0), to slightly $(+1)$, moderately $(+2)$, and markedly improved $(+3)$. Patients with moderately and markedly improved results after treatment were considered to have successful treatment outcomes. Otherwise, the treatment was considered to have failed.

\section{Urodynamic Studies}

All patients underwent a comprehensive videourodynamic study with a Urolab Janus 6 device (LifeTech, Inc., Stafford, Texas, USA) using a double lumen $6 \mathrm{Fr}$ catheter by one examiner in an identically aseptic manner. The pressure flow study was performed using the standard procedure in a sitting position according to the International Continence Society standardization (24). After the videourodynamic studies, $40 \mathrm{~mL}$ of $0.4 \mathrm{M} \mathrm{KCl}$ solution was infused slowly into the bladder and the test was regarded as positive when painful (increased VAS score $\geq 2$ ) or urgency sensation was elicited compared to normal saline infusion during prior urodynamic study (25).

This study was approved by the ilRB and ethics committee of the hospital. Each patient was informed about the study rationale and procedures; written, informed consent was obtained before treatment.

\section{Botulinum Toxin Injections and Follow-up}

The BoNT-A injection was performed in the operating room. Patients received an intravesical injection of $100 \mathrm{U}$ of BoNT-A (onabtulinumtoxinA, Allergan, Irvine, CA, USA) followed by cystoscopic hydrodistention under intravenous general anesthesia. Each vial of BoNT-A was diluted with $20 \mathrm{ml}$ of normal saline and 40 suburothelial injections were made. The injection needle was inserted into the urothelium at the posterior and lateral walls of the bladder, using a 23-gauge needle and rigid cystoscopic injection instrument (22 Fr, Richard Wolf, Knittlingen, Germany). Cystoscopic hydrodistention was performed to an intravesical pressure of $80 \mathrm{~cm}$ of water for 15 minutes and the maximal bladder capacity (MBC) under hydrodistention was recorded. When glomerulations, petechia, and mucosal fissure developed after bladder deflation, they were graded from 0 to 4 , indicating none, mild, moderate, severe grade, and ulceration, respectively (19). Bladder biopsies were taken at 4 sites about $2 \mathrm{~cm}$ lateral and posterior to the ureteral orifice immediately after hydrodistention.

After the BoNT-A injections, a 14-Fr urethral Foley catheter remained for one day and patients were discharged on the next day. Oral antibiotics were pre- scribed for 7 days. Patients were monitored in the outpatient clinic 2 weeks later. Analgesic or tricyclic antidepressant medications were continued as before treatment.

The patients were monitored in the out-patient clinic every 3 months. During each follow-up visit, data from the 3-day voiding diary and symptom inventory using the O'Leary-Sant symptom scores (OSS), pain VAS, and GRA were recorded. The largest voided volume in the 3-day voiding diary was considered the measure of functional bladder capacity (FBC). At 6 months after the initial BoNT-A injection, patients were questioned about bladder condition. Repeat BoNT-A injection and hydrodistention was recommended 6 months after the initial treatment if patients felt a relapse of baseline symptoms or desired repeated treatment to achieve a better treatment outcome. The BoNT-A injection was repeated every 6 months up to 4 times or until patients declared their symptoms had significantly resolved or patients did not want repeated treatment because of lack of efficacy or adverse events.

Videourodynamic studies were performed at baseline and 6 months after each BoNT-A treatment. The urodynamic parameters included first sensation of bladder filling, first desire to void, strong desire to void, cystometric bladder capacity (CBC), detrusor pressure, maximum flow rate during voiding, and postvoid residual. The $\mathrm{KCl}$ test was also performed after each urodynamic study.

\section{Statistical Analysis}

The results of the 3-day voiding diary, urodynamic studies, ICSI, ICPI, and pain VAS were compared between baseline and each treatment time point. Continuous variables are presented as means \pm standard deviations (SDs), and categorical data are presented as numbers and percentages (\%). Statistical comparisons between the groups were tested using the chi-square test for categorical variables and the Wilcoxon ranksum test for continuous variables. Repeated measurement analysis was used for analysis of variables at different time points. Long-term successful results were compared using Kaplan-Meier analyses. Statistical assessments were considered significant when $P<0.05$. Statistical analyses were performed using SPSS 15.0 statistical software (SPSS Inc., Chicago, IL).

\section{Results}

A total of 81 patients (71 women and 10 men, mean age 48 and 48.2 years, respectively) entered 
this study and received the first time BoNT-A injection. At 6 months after the initial BoNT-A injection, patients could decide whether they wanted to continue BoNT-A injection or not, based on their subjective therapeutic effects. Table 1 shows the patients' status and therapeutic effects of initial BoNT-A injection. After the first, second, and third injections, 6,9 , and 8 patients felt great improvement and did not want to continue with injections. In the meantime, 14, 10, and 4 patients felt symptoms did not improve and did not receive further injections. Overall, 81 patients after the first injection, 61 after the second injection, 42 after the third injection, and 30 after the fourth injection were available for followup. There were 26 women and 4 men who completed 4 injections and follow-up; their mean age was $45 \pm$ 23 years. The median duration of IC/PBS was 5 years (range, 3 to 23 years).

Table 2 lists the variables included in the GRA, symptom scores, VAS, voiding diary, uroflowmetry, and $\mathrm{MBC}$ at baseline of each injection time point. The data include patients who have improved or failed treatment outcome at the baseline of each injection time-point. Although most of the symptom, pain, and bladder capacity variables showed no significant difference among patients receiving 1, 2, and 3 injections, all

Table 1. The patients' status of repeated BoNT-A injection based on the therapeutic effects at 6 months after BoNT-A injection and patient's will of continued treatment

\begin{tabular}{|l|c|c|c|c|c|}
\hline \hline Months After & N & Improved \& Continue & Ineffective \& Continue & Improved \& Discontinue & Ineffective \& Discontinue \\
\hline BoNT-A X1 & 81 & $\begin{array}{c}48 \\
(59.3 \%)\end{array}$ & $\begin{array}{c}13 \\
(16.0 \%)\end{array}$ & $\begin{array}{c}6 \\
(7.4 \%)\end{array}$ \\
\hline BoNT-A X2 & 61 & $\begin{array}{c}31 \\
(50.8 \%)\end{array}$ & $\begin{array}{c}11 \\
(18.0 \%)\end{array}$ & $\begin{array}{c}1 \\
(14.8 \%)\end{array}$ \\
\hline BoNT-A X3 & 42 & $\begin{array}{c}22 \\
(52.4 \%)\end{array}$ & $\begin{array}{c}8 \\
(19.0 \%)\end{array}$ & $\begin{array}{c}10 \\
(16.4 \%)\end{array}$ \\
\hline BoNT-A X4 & 30 & $\begin{array}{c}10 \\
(33.3 \%)\end{array}$ & $\begin{array}{c}5 \\
(16.7 \%)\end{array}$ & $\begin{array}{c}13 \\
(9.5 \%)\end{array}$ \\
\hline
\end{tabular}

BoNT-A: onabotulinumtoxinA

Table 2. Changes of baseline parameters before each time-point of BoNT-A injection.

\begin{tabular}{|c|c|c|c|c|c|}
\hline & $\begin{array}{c}\text { BoNT-A x1 } \\
\text { Baseline }(\mathrm{N}=\mathbf{8 1})\end{array}$ & $\begin{array}{c}\text { BoNT-A x2 } \\
\text { Baseline }(\mathrm{N}=61)\end{array}$ & $\begin{array}{c}\text { BoNT-A x } 3 \\
\text { Baseline }(\mathrm{N}=42)\end{array}$ & $\begin{array}{c}\text { BoNT-A x } 4 \\
\text { Baseline }(\mathrm{N}=30)\end{array}$ & $P$ value \\
\hline ICSI & $12.1 \pm 3.2$ & $8.84 \pm 4.39$ & $9.54 \pm 4.31$ & $8.88 \pm 4.16$ & $<0.0001$ \\
\hline ICPI & $11.1 \pm 2.98$ & $7.95 \pm 4.43$ & $8.39 \pm 4.59$ & $7.09 \pm 4.84$ & $<0.0001$ \\
\hline OSS & $23.2 \pm 5.87$ & $19.8 \pm 8.51$ & $17.9 \pm 8.68$ & $15.9 \pm 8.81$ & $<0.0001$ \\
\hline VAS & $5.13 \pm 2.32$ & $3.60 \pm 2.34$ & $3.90 \pm 2.58$ & $3.22 \pm 2.55$ & $<0.0001$ \\
\hline FBC & $135 \pm 77.9$ & $170 \pm 82.8$ & $197 \pm 105$ & $223 \pm 119$ & $<0.0001$ \\
\hline Frequency & $13.8 \pm 4.77$ & $10.6 \pm 5.12$ & $11.8 \pm 6.24$ & $10.9 \pm 5.91$ & 0.110 \\
\hline Nocturia & $4.25 \pm 3.83$ & $3.06 \pm 1.69$ & $3.39 \pm 2.45$ & $3.59 \pm 2.94$ & 0.507 \\
\hline Qmax & $14.1 \pm 4.69$ & $13.0 \pm 6.03$ & $13.2 \pm 6.40$ & $12.2 \pm 6.10$ & 0.526 \\
\hline Volume & $253 \pm 112$ & $184 \pm 65.2$ & $207 \pm 91.8$ & $202 \pm 82.3$ & 0.002 \\
\hline PVR & $14 \pm 35.7$ & $29 \pm 53.3$ & $48 \pm 86.9$ & $46 \pm 66$ & 0.335 \\
\hline СBC & $270 \pm 112$ & $302 \pm 116$ & $352 \pm 134$ & $321 \pm 160$ & 0.008 \\
\hline $\mathrm{MBC}$ & $667 \pm 216$ & $728 \pm 196$ & $739 \pm 216$ & $724 \pm 204$ & 0.531 \\
\hline GRA & 0 & $1.38 \pm 1.16$ & $1.50 \pm 0.76$ & $1.69 \pm 1.26$ & $<0.0001$ \\
\hline
\end{tabular}

BoNT-A: onabotulinumtoxinA, CBC: cystometric bladder capacity, FBC: functional bladder capacity, GRA: global response assessment, ICPI: interstitial cystitis problem indexes, ICSI: interstitial cystitis symptom indexes, MBC: maximal bladder capacity, OSS: O'Leary-Sant symptom score, PVR: postvoid residual, Qmax: maximum flow rate, VAS: visual analog scale 
showed significant improvement compared to baseline. The GRA has a trend to increase with increasing injection number.

Table 3 shows the measured variables at all timepoints in patients who received 4 BoNT-A injections. The OSS $(24.8 \pm 6.18 \vee 15.2 \pm 8.38, P<0.0001)$, VAS $(5.8 \pm 2.27$ v $3.0 \pm 2.3, P<0.0001), \mathrm{FBC}(139 \pm 81.2 \mathrm{v}$ $228 \pm 118, P<0.0001)$, and daytime frequency $(13.8 \pm$ $5.1 \vee 10.3 \pm 4.94, P=0.029$ ) all showed significant improvement after 4 repeated treatments. Although the glomerulation grade $(2.5 \pm 1.3 \vee 1.2 \pm 1.1, P<0.0001)$ showed significant improvement, MBC $(711 \pm 217 \mathrm{v}$ $739 \pm 186, P=0.472$ ) did not improve significantly after 4 BoNT-A injections. The $\mathrm{KCl}$ test was positive in all patients at baseline and turned negative in 12 (40\%) patients at baseline of the fourth BoNT-A treatment. Overall, the GRA and symptom variables showed persistent improvement with each repeated treatment when baseline of each successive treatment was compared with the initial study baseline level.

The adverse events are listed in Table 4. About $30 \%$ of patients had dysuria after each BoNT-A injection; nevertheless, only one episode of acute urinary retention occurred and only one patient needed clean intermittent self-catheterization for 3 months. UTI developed in $4.9 \%$ to $19 \%$ of patients after each BoNT-A treatment. The occurrence of adverse events did not increase with increasing number of BoNT-A injections $(P=0.235)$.

At 6 months after the fourth BoNT-A injection, 23 $(76.6 \%)$ of 30 patients had improvements in GRA by 2 points compared to their baseline conditions. Five patients with an ineffective result and 10 with an improved result and 5 with an ineffective outcome requested to continue the BoNT-A injection to achieve a better long-term outcome. Among the 30 patients receiving 4 repeated BoNT-A injections, bladder pain improved in $27(90 \%)$ and frequency urgency improved in $21(70 \%)$. All 21 patients with improvement in frequency urgency also had bladder pain improvement. Among the 20,19, and 12 patients who discontinued the treatment after the first, second, and third BoNT-A injections, $6(30 \%), 14(63.5 \%)$, and $9(75 \%)$ remained satisfied with their results, respectively, after followup for 12 months. Kaplan-Meier survival curves among patient subgroups with different numbers of BoNT-A injections revealed better success rates in the patients who received more than one injection $(P=0.0404)$ and significantly better results in patients who had 4 repeated injections $(P=0.0242)$ and 3 injections $(P=$ 0.050), compared to those who received a single injection. However, there was no significant difference of long-term success rates among patients who received 2,3 , and 4 injections (all $P>0.05$ ) (Fig.1).

Table 3. Changes of parameters in the 30 patients receiving 4 repeated onabotulinumtoxinA injections.

\begin{tabular}{|l|c|c|c|c|c||}
\hline $\begin{array}{l}\text { BoNT-A x 4 } \\
(\text { N=30 })\end{array}$ & $\begin{array}{c}\text { BoNT-A xI } \\
\text { Baseline }\end{array}$ & $\begin{array}{c}\text { BoNT-A x2 } \\
\text { Baseline }\end{array}$ & $\begin{array}{c}\text { BoNT-A x3 } \\
\text { Baseline }\end{array}$ & $\begin{array}{c}\text { BoNT-A x 4 } \\
\text { Baseline }\end{array}$ & P value \\
\hline ICSI & $12.9 \pm 3.47$ & $9.27 \pm 4.55$ & $8.9 \pm 3.66$ & $8.57 \pm 3.94$ & $<0.0001$ \\
\hline ICPI & $11.8 \pm 3.0$ & $8.8 \pm 4.47$ & $8.2 \pm 4.13$ & $6.63 \pm 4.6$ & $<0.0001$ \\
\hline OSS & $24.8 \pm 6.18$ & $18.1 \pm 8.68$ & $17.1 \pm 7.57$ & $15.2 \pm 8.38$ & $<0.0001$ \\
\hline VAS & $5.8 \pm 2.27$ & $4.03 \pm 2.34$ & $3.70 \pm 2.35$ & $3.03 \pm 2.3$ & $<0.0001$ \\
\hline FBC & $139 \pm 81.2$ & $170 \pm 87.6$ & $210 \pm 100$ & $228 \pm 118$ & $<0.0001$ \\
\hline Frequency & $13.8 \pm 5.1$ & $11.3 \pm 6.31$ & $11.5 \pm 5.93$ & $10.3 \pm 4.94$ & 0.029 \\
\hline Nocturia & $3.73 \pm 2.12$ & $3.13 \pm 1.85$ & $3.13 \pm 2.33$ & $3.4 \pm 2.79$ & 0.384 \\
\hline Qmax & $14.4 \pm 4.95$ & $13.5 \pm 7.13$ & $13.6 \pm 4.99$ & $12.4 \pm 6.22$ & 0.535 \\
\hline Volume & $252 \pm 111$ & $279 \pm 144$ & $296 \pm 137$ & $283 \pm 150$ & 0.289 \\
\hline PVR & $9.84 \pm 21.9$ & $41.2 \pm 67.4$ & $38.8 \pm 82.3$ & $47.3 \pm 66.7$ & 0.127 \\
\hline CBC & $262 \pm 116$ & $323 \pm 126$ & $338 \pm 113$ & $335 \pm 145$ & 0.009 \\
\hline MBC & $711 \pm 217$ & $752 \pm 218$ & $756 \pm 202$ & $739 \pm 186$ & 0.472 \\
\hline GRA & 0 & $1.40 \pm 1.19$ & $1.53 \pm 0.78$ & $1.77 \pm 1.25$ & $<0.0001$ \\
\hline
\end{tabular}

BoNT-A: onabotulinumtoxinA, CBC: cystometric bladder capacity, FBC: functional bladder capacity, GRA: global response assessment, ICPI: interstitial cystitis problem indexes, ICSI: interstitial cystitis symptom indexes, MBC: maximal bladder capacity, OSS: O'Leary-Sant symptom score, PVR: postvoid residual, Qmax: maximum flow rate, VAS: visual analog scale 
Pain Physician: January/February 2013; 16:E15-E23

Table 4. Adverse events occurred after repeated BoNT-A injections, no significant difference among each injection group $(P=0.235)$.

\begin{tabular}{|c|c|c|c|c|c|c|c|}
\hline & \multicolumn{7}{|c|}{ Adverse events } \\
\hline & None & UTI & Dysuria & CISC & AUR & Hematuria & Total \\
\hline BoNT-A X 1 & $\begin{array}{c}52 \\
(64.2 \%)\end{array}$ & $\begin{array}{c}4 \\
(4.9 \%)\end{array}$ & $\begin{array}{c}23 \\
(28.4 \%)\end{array}$ & 0 & 0 & $\begin{array}{c}2 \\
(2.5 \%)\end{array}$ & 81 \\
\hline BoNT-A X 2 & $\begin{array}{c}33 \\
(54.1 \%)\end{array}$ & $\begin{array}{c}5 \\
(8.2 \%) \\
\end{array}$ & $\begin{array}{c}21 \\
(34.4 \%)\end{array}$ & 0 & $\begin{array}{c}1 \\
(1.6 \%)\end{array}$ & $\begin{array}{c}1 \\
(1.8 \%)\end{array}$ & 61 \\
\hline BoNT-A X 3 & $\begin{array}{c}18 \\
(42.9 \%)\end{array}$ & $\begin{array}{c}8 \\
(19.0 \%)\end{array}$ & $\begin{array}{c}15 \\
(35.7 \%)\end{array}$ & $\begin{array}{c}1 \\
(2.4 \%)\end{array}$ & 0 & 0 & 42 \\
\hline BoNT-A X 4 & $\begin{array}{c}19 \\
(63.3 \%)\end{array}$ & $\begin{array}{c}2 \\
(6.7 \%)\end{array}$ & $\begin{array}{c}9 \\
(30.0 \%)\end{array}$ & 0 & 0 & 0 & 30 \\
\hline
\end{tabular}

AUR: acute urinary retention, BoNT-A: onabotulinumtoxinA, CISC: clean intermittent self-catheterization, UTI: urinary tract infection

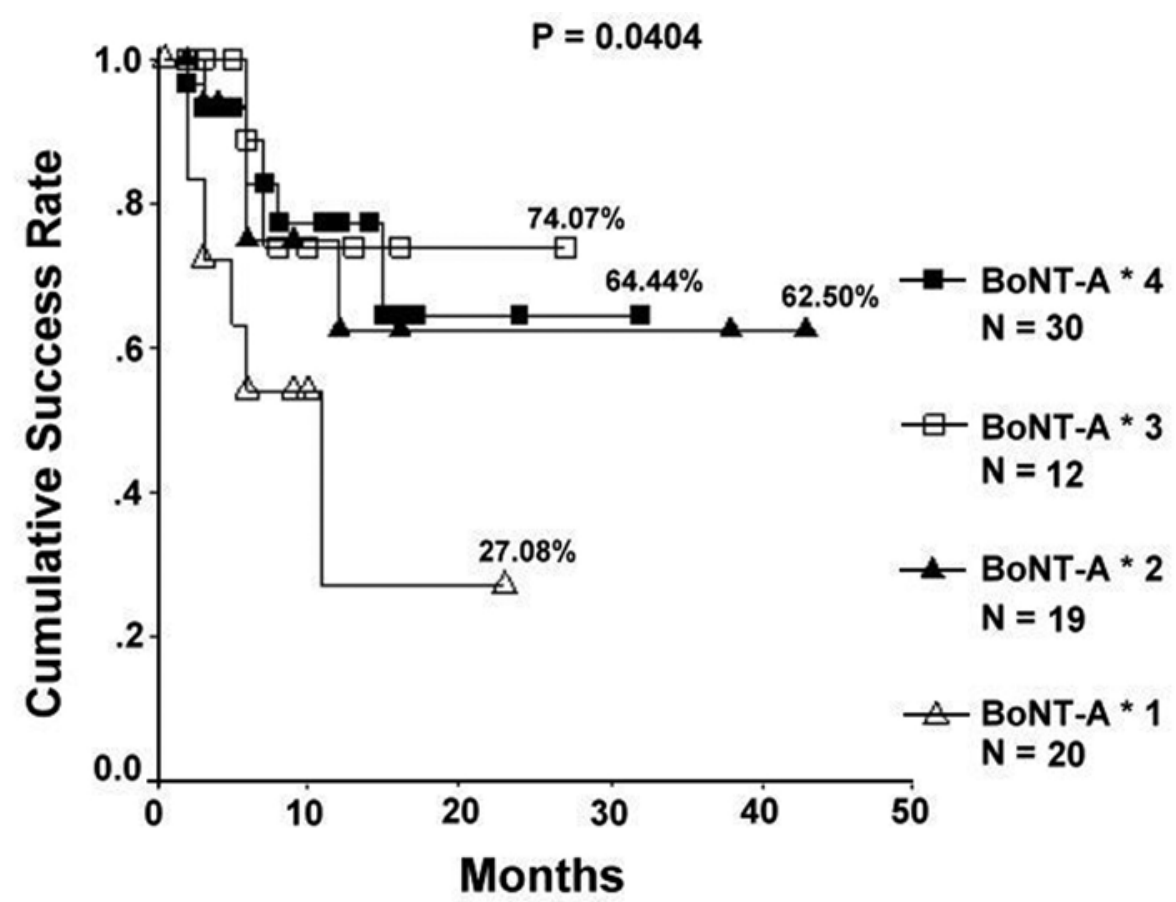

Fig. 1. The cumulative success rates of teh 81 patients receiving single injection or different numbers of repeated onabotulinumtoxin $A$ (BoNT-A) injections.

\section{Discussion}

The results of this study demonstrated that repeated intravesical injections of BoNT-A increase FBC and provided long-term pain relief in patients with IC/PBS who were refractory to conventional treatment. The long-term success rates of 3 or 4 repeated intravesical BoNT-A injections were better than a single injection. These therapeutic effects might involve not only inhibiting release of acetylcholine in the neuromuscular junctions of the detrusor, but also an anti-inflammatory response.

Currently, there is no satisfactory treatment for IC/PBS. Although a leaky urothelium is speculated to cause chronic inflammation of the bladder, intravesical heparin therapy or oral pentosanpolysulphate cannot eradicate the bladder pain and intractable frequency in most patients with IC/PBS $(23,26)$, suggesting restora- 
tion of epithelial integrity can only partially repair the damaged urothelial barrier, but not the submucosal inflammation or possible central sensitization pain process that characterizes IC/PBS. Searching for a treatment modality for long-term effect on IC/PBS is mandatory.

Review of previous investigations of BoNT-A on IC/PBS shows variable results. Smith et al (14) reported a $67 \%$ success rate with a therapeutic duration of 9 months. Giannantoni et al (10) reported $85.7 \%$ of patients had an initial improvement, but the duration was only 3 months. In their long-term follow-up study, the therapeutic effect decreased to $26.6 \%$ at 5 months and none had any effect at 12 months after treatment (18). Our recent study revealed that single intravesical injections of BoNT-A followed by hydrodistention produced significantly better clinical results than hydrodistention alone (27). A recent large-cohort study revealed that the IC/PBS symptom and pain score and FBC were significantly improved in patients who underwent hydrodistention and 100U BoNT-A injection (7). Intravesical BoNT-A injection appears to be a safe and effective therapeutic option for analgesia and increased bladder capacity for patients with IC/PBS. In the current clinical trial of repeated BoNT-A injections, a higher success rate with a longer therapeutic duration occurred in patients receiving more than 2 injections, which was expected.

The relationship between urothelial apoptosis and chronic bladder inflammation in the bladder wall of IC/ PBS and after intravesical BoNT-A injections was investigated recently (28). A single BoNT-A injection improved clinical symptoms, pain score, and daytime frequency in 23 patients. In the histological analysis, mast cell activity stain and apoptotic cell count did not decrease significantly, Bax and p-p38 but not tryptase content showed significant decrease after a single BoNT-A injection. However, in the 11 patients receiving 3 repeated BoNT-A injections, significant decrease of pain score, glomerulation degree, and GRA were noted after treatment. The tryptase, Bax, p-p38 contents, and apoptotic cell counts all showed significant decrease. SNAP25 content in the bladder also decreased after BoNT-A treatments, which confirmed the therapeutic effect of repeated BoNT-A injections. This study confirms that chronic inflammation and apoptotic signaling molecules can be significantly reduced after repeated BoNT-A injections in IC/PBS bladders, suggesting repeated BoNT-A injections is necessary to achieve a higher success rate in treatment of IC/PBS.

BoNT-A also has an anti-inflammatory effect on cystitis in rats (14). It reduces bladder nerve growth factor (NGF) levels after injection in IC/PBS patients, yield- ing satisfactory pain relief (29). A significant, transient reduction in urinary NGF and brain derived NGF was also observed in IC/PBS patients after tigonal BoNT-A injection (30). This study further confirms that pain relief in IC/PBS patients after repeated BoNT-A injection is more prominent than relief of frequency urgency. This result reveals that the bladder pain and bladder hypersensitivity in IC/BPS bladders could be through different pathways. Therefore, although bladder pain is reduced after repeated BoNT-A injections, the bladder still remains hypersensitive in some patients, which could be caused by unresolved bladder inflammation.

One interesting finding after repeated BoNT-A injection was the decreased grade of glomerulations, without an increase in MBC after hydrodistention. Increased apoptosis of vascular endothelial cells was shown in the bladder of IC/PBS patients (31). Vascular epidermal growth factor overexpression is responsible for the glomerulations in IC/PBS (32). The decrease in glomerulations after repeated BoNT-A injections in our study is likely due to improvement of chronic bladder inflammation. However, patients might have improved glomerulations without increased $\mathrm{MBC}$, or have increased MBC without improvement of glomerulations. Furthermore, although daytime frequency and FBC improved after repeated BoNT-A injections, nocturia did not show significant improvement. These phenomena revealed that the downstream changes of chronic inflammation in IC/PBS might be through multiple pathophysiologic pathways. Four repeated BoNT-A injections might not achieve complete resolution of bladder inflammation in some patients. Increased injection numbers or increased BoNT-A dose should be considered in patients refractory to repeated BoNT-A treatments.

\section{Limitations}

The most important limitations of this study are the lack of a control arm and that patient allocation was not randomized. Due to ethical considerations, a placebo control was not allowed by the IRB. However, the use of subjects receiving only one injection compared to those receiving 4 injections did provide some valuable insight. Although patients were treated based on their subjective treatment outcome, the long-term effects on pain improvement and objective increase of bladder capacity provide evidence for the success of repeated BoNT-A injections. We hope more comprehensive immunohistochemical studies of bladder biopsies provide evidence for the therapeutic effects of repeated BoNT-A treatment. 


\section{Conclusion}

Repeated intravesical BoNT-A injections were safe and effective for pain relief and they increased bladder capacity and provided a better long-term success rate than a single injection did for treatment of IC/PBS.

\section{References}

1. Hanno PM, Sant GR. Clinical highlights of the national Institute of Diabetes and Digestive and Kidney Diseases/Interstitial Cystitis Association scientific conference on interstitial cystitis. Urology 2001; 57:2-6.

2. Parsons $\mathrm{CL}$. The role of the urinary epithelium in the pathogenesis of interstitial cystitis/prostatitis/urethritis. Urology 2007; 69:9-16.

3. Shie JH, Kuo HC. Higher levels of cell apoptosis and abnormal E-cadherin expression in the urothelium are associated with inflammation in patients with interstitial cystitis/painful bladder syndrome. BJU Int 2011; 108: E136-E141.

4. Brady CM, Apostolidis AN, Harper M, Yiangou $Y$, Beckett A, Jacques TS, Freeman A, Scaravilli F, Fowler C), Anand P. Parallel changes in bladder suburothelial vanilloid receptor TRPV 1 and panneuronal marker PGP9.5 immunoreactivity in patients with neurogenic detrusor overactivity after intravesical resiniferatoxin treatment. BJU Int 2004; 93:770-776.

5. Cockayne DA, Hamilton SG, Zhu QM, Dunn PM, Zhong Y, Novakovic $S$, Malmberg AB, Cain G, Berson A, Kassotakis L, Hedley L, Lachnit WG, Burnstock G, McMahon SB, Ford AP. Urinary bladder hyporeflexia and reduced pain-related behaviour in $\mathrm{P}_{2} \mathrm{X}_{3}$-deficient mice. Nature 2000; 407:1011-1015.

6. Cayan S, Coskun B, Bozlu M, Acar D, Akbay $E$, Ulusoy E. Botulinum toxin type $A$ may improve bladder function in a rat chemical cystitis model. Urol Res 2003; 30:399-404.

7. Chung SD, Kuo YC, Kuo HC. Intravesical onabotulinumtoxinA injections for refractory painful bladder syndrome. Pain Physician 2012; 15:197-202.

8. Rapp DE, Turk KW, Bales GT, Cook SP. Botulinum toxin type a inhibits calcitonin gene-related peptide release from isolated rat bladder. J Urol 2006; 175:1138-1142.

9. Chuang YC, Yoshimura N, Huang CC, Chiang PH, Chancellor MB. Intravesical botulinum toxin A administration produces analgesia against acetic acid in- duced bladder pain response in rats. J Urol 2004; 172:1529-1532.

10. Giannantoni A, Di Stasi SM, Nardicchi V, Zucchi A, Macchioni L, Bini V, Goracci G, Porena M. Botulinum-A toxin injections into the detrusor muscle decrease nerve growth factor bladder tissue levels in patients with neurogenic detrusor overactivity. J Urol 2006; 175:2341-2344.

11. Khera M, Somogyi GT, Kiss S, Boone TB, Smith CP. Botulinum toxin A inhibits ATP release from bladder urothelium after chronic spinal cord injury. Neurochem Int 2004; 45:987-993.

12. Apostolidis A, Papat R, Yiangou Y, Cockayne D, Ford AP, Davis JB, Dasgupta P, Fowler CJ, Anand P. Decreased sensory receptors $\mathrm{P}_{2} \mathrm{X}_{3}$ and $T R P \mathrm{~V}_{1}$ in suburothelial nerve fibers following intradetrusor injection of botulinum A toxin for human detrusor overactivity. J Urol 2005; 174:977-982.

13. Reitz A, Stöhrer M, Kramer G, Del Popolo G, Chartier-Kastler E, Pannek J, Burgdörfer $H$, Göcking K, Madersbacher H, Schumacher S, Richter R, von Tobel J, Schurch B. European experience of 200 cases treated with botulinum-A toxin injections into the detrusor muscle for urinary incontinence due to neurogenic detrusor overactivity. Eur Urol 2004; 45:510-515.

14. Smith CP, Radziszewski P, Borkowski A, Somogyi GT, Boone TB, Chancellor MB. Botulinum toxin A has antinociceptive effects in treating interstitial cystitis. Urology 2004; 64:871-875.

15. Kuo HC. Preliminary results of suburothelial injection of botulinum A toxin in the treatment of chronic interstitial cystitis. Urol Int 2005; 75:170-174.

16. Giannantoni A, Costantini E, Di Stasi SM, Tascini MC, Bini V, Porena M. Botulinum $A$ toxin intravesical injections in the treatment of painful bladder syndrome: A pilot study. Eur Urol 2006; 49:704-709.

17. Cui $M$, Aoki KR. Botulinum toxin type A (BTX-A) reduces inflammatory pain in the rat formalin model. Cephalalgia 2000; 20:414-418.

18. Giannantoni A, Porena M, Costantini E, Zucchi A, Mearini L, Mearini E. Botuli- num $A$ toxin intravesical injection in patients with painful bladder syndrome: 1-year followup. J Urol 2008;179:10311034.

19. Homma $\mathrm{Y}$, Ueda $\mathrm{T}$, Tomoe H, Lin AT, Kuo HC, Lee MH, Lee JG, Kim DY, Lee $\mathrm{KS}$; Interstitial cystitis guideline committee. Clinical guidelines for interstitial cystitis and hypersensitive bladder syndrome. Int J Urol 2009; 16:597-615.

20. NIDDK Workshop of Interstitial Cystitis. Besthesda, Maryland, USA, Aug 28-29, 1987.

21. Lubeck DP, Whitmore K, Sant GR, Alvarez-Horine S, Lai C. Psychometric validation of the O'Leary-Sant interstitial cystitis symptom index in a clinical trial of pentosan polysulfate sodium. Urology 2001; 57:62-66.

22. Nickel JC, Barkin J, Forrest J, Mosbaugh PG, Hernandez-Graulau J, Kaufman D, Lloyd K, Evans RJ, Parsons CL, Atkinson LE; Elmiron Study Group. Randomized, double-blind, dose-ranging study of pentosan polysulfate sodium for interstitial cystitis. Urology 2005; 65:654-658.

23. Sant GR, Propert KJ, Hanno PM, Burks D, Culkin D, Diokno AC, Hardy C, Landis JR, Mayer R, Madigan R, Messing EM, Peters K, Theoharides TC, Warren J, Wein AJ, Steers W, Kusek JW, Nyberg LM; Interstitial Cystitis Clinical Trials Group. A pilot clinical trial of oral pentosan polysulphate and oral hydroxyzine in patients with interstitial cystitis. J Urol 2003; 170:810-815.

24. Abrams P, Cardozo L, Fall M, Griffiths D Rosier $P$, Ulmsten $U$, van Kerrebroeck $P$, Victor A, Wein A; Standardisation Subcommittee of the International Continence Society. The standardisation of terminology of lower urinary tract function: Report from the Standardisation Sub-committee of the International Continence Society. Neurourol Urodyn 2002; 21:167-178.

25. Parsons $\mathrm{CL}$. The potassium sensitivity test: A new gold standard for diagnosing and understanding the pathophysiology of interstitial cystitis. J Urol 2009; 182:432-434.

26. Parsons CL, Housley T, Schmidt JD, Leb- 
ow D. Treatment of interstitial cystitis with intravesical heparin. $\mathrm{Br}$ J Urol 1994; 73:504-507.

27. Kuo HC, Chancellor MB. Comparison of intravesical botulinum toxin type $A$ injections plus hydrodistention with hydrodistention alone for the treatment of refractory interstitial cystitis/painful bladder syndrome. BJU Int 2009; 104:657-661.

28. Shie JH, Liu HT, Wang YS, Kuo HC. Immunohistochemical evidence suggests repeated intravesical application of botulinum toxin $\mathrm{A}$ injections may improve treatment efficacy of interstitial cystitis/bladder pain syndrome. BJU Int 2012 (ePub ahead of Print)

29. Liu HT, Kuo HC. Intravesical botulinum toxin $\mathrm{A}$ injections plus hydrodistension can reduce nerve growth factor production and control bladder pain in interstitial cystitis. Urology 2007; 70:463-468.

30. Pinto R, Lopes T, Frias B, Silva A, Silva JA, Silva CM, Cruz C, Cruz F, Dinis P. Trigonal injection of botulinum toxin $A$ in patients with refractory bladder pain syndrome/interstitial cystitis. Eur Urol 2010; 58:360-365.
31. Yamada T, Nishimura M, Mita H. Increased number of apoptotic endothelial cells in bladder of interstitial cystitis patients. World J Urol 2007; 25:407-413.

32. Kiuchi H, Tsujimura A, Takao T, Yamamoto K, Nakayama J, Miyagawa Y, Nonomura N, Takeyama M, Okuyama A. Increased vascular endothelial growth factor expression in patients with bladder pain syndrome/ interstitial cystitis: Its association with pain severity and glomerulations. BJU Int 2009; 104:826-831. 
